\title{
Rate of Convergence of Modified Baskakov-Durrmeyer Type Operators for Functions of Bounded Variation
}

\author{
Prashantkumar Patel ${ }^{1,2}$ and Vishnu Narayan Mishra ${ }^{1,3}$ \\ ${ }^{1}$ Department of Applied Mathematics \& Humanities, Sardar Vallabhbhai National Institute of Technology, \\ Surat, Gujarat 395 007, India \\ ${ }^{2}$ Department of Mathematics, St. Xavier's College, Ahmedabad, Gujarat 380 009, India \\ ${ }^{3}$ L. 1627 Awadh Puri Colony Beniganj, Phase-III, Opposite-Industrial Training Institute (ITI), Ayodhya Main Road, \\ Faizabad, Uttar Pradesh 224 001, India \\ Correspondence should be addressed to Prashantkumar Patel; prashant225@gmail.com
}

Received 19 April 2014; Accepted 11 June 2014; Published 2 July 2014

Academic Editor: Abdelalim A. Elsadany

Copyright (C) 2014 P. Patel and V. N. Mishra. This is an open access article distributed under the Creative Commons Attribution License, which permits unrestricted use, distribution, and reproduction in any medium, provided the original work is properly cited.

We study a certain integral modification of well-known Baskakov operators with weight function of beta basis function. We establish rate of convergence for these operators for functions having derivative of bounded variation. Also, we discuss Stancu type generalization of these operators.

\section{Introduction}

The integral modification of Baskakov operators having weight function of some beta basis function are defined as the following: for $x \in[0, \infty), \gamma>0$,

$$
\begin{aligned}
B_{n, \gamma}(f, x)= & \sum_{k=1}^{\infty} p_{n, k, \gamma}(x) \int_{0}^{\infty} b_{n, k, \gamma}(t) f(t) d t \\
& +(1+\gamma x)^{-n} f(0) \\
= & \int_{0}^{\infty} W_{n, \gamma}(x, t) f(t) d t,
\end{aligned}
$$

where

$$
\begin{aligned}
& p_{n, k, \gamma}(x)=\frac{\Gamma(n / \gamma+k)}{\Gamma(k+1) \Gamma(n / \gamma)} \cdot \frac{(\gamma x)^{k}}{(1+\gamma x)^{(n / \gamma)+k},} \\
& b_{n, k, \gamma}(t)=\frac{\gamma \Gamma(n / \gamma+k+1)}{\Gamma(k) \Gamma(n / \gamma+1)} \cdot \frac{(\gamma t)^{k-1}}{(1+\gamma t)^{(n / \gamma)+k+1}},
\end{aligned}
$$

$$
W_{n, \gamma}(x, t)=\sum_{k=1}^{\infty} p_{n, k, \gamma}(x) \int_{0}^{\infty} b_{n, k, \gamma}(t) d t+(1+\gamma x)^{-n / \gamma} \delta(t)
$$

$\delta(t)$ being the Dirac delta function.

The operators defined by (1) were introduced by Gupta [1]; these operators are different from the usual BaskakovDurrmeyer operators. Actually these operators satisfy condition $B_{n, \gamma}(a t+b, x)=a x+b$, where $a$ and $b$ are constants. In [1], the author estimated some direct results in simultaneous approximation for these operators (1). In particular case $\gamma=$ 1 , the operators (1) reduce to the operators studied in $[2,3]$.

In recent years a lot of work has been done on such operators. We refer to some of the important papers on the recent development on similar type of operators [4-9]. The rate of convergence for certain Durrmeyer type operators and the generalizations is one of the important areas of research in recent years. In present article, we extend the studies and here we estimate the rate of convergence for functions having derivative of bounded variation. 
We denote $\phi_{n, \gamma}(x, t)=\int_{0}^{t} W_{n, \gamma}(x, s) d s$; then, in particular, we have

$$
\phi_{n, \gamma}(x, \infty)=\int_{0}^{\infty} W_{n, \gamma}(x, s) d s=1
$$

By $D B_{r}(0, \infty), r \geq 0$ we denote the class of absolutely continuous functions $f$ defined on the interval $(0, \infty)$ such that,

(i) $f(t)=O\left(t^{r}\right), t \rightarrow \infty$.

(ii) having a derivative $f^{\prime}$ on the interval $(0, \infty)$ coinciding a.e. with a function which is of bounded variation on every finite subinterval of $(0, \infty)$.

It can be observed that all function $f \in B D_{r}(0, \infty)$ possess for each $c>0$ a representation

$$
f(x)=f(c)+\int_{c}^{x} \psi(t) d t, \quad x \geq c .
$$

\section{Rate of Convergence for $B_{n, \gamma}$}

Lemma 1 (see [1]). Let the function $T_{n, m, \gamma}(x), m \in \mathbb{N} \cup\{0\}$, be defined as

$$
\begin{aligned}
T_{n, m, \gamma}(x)= & B_{n, \gamma}\left((t-x)^{m}, x\right) \\
= & \sum_{k=1}^{\infty} p_{n, k, \gamma}(x) \int_{0}^{\infty} b_{n, k, \gamma}(t)(t-x)^{m} d t \\
& +(1+\gamma x)^{-n / \gamma}(-x)^{m} .
\end{aligned}
$$

Then it is easily verified that, for each $x \in(0, \infty), T_{n, 0, \gamma}(x)=1$, $T_{n, 1, \gamma}(x)=0$, and $T_{n, 2, \gamma}(x)=2 x(1+\gamma x) /(n-\gamma)$, and also the following recurrence relation holds:

$$
\begin{aligned}
(n-\gamma m) T_{n, m+1, \gamma}(x)= & x(1+\gamma x) \\
& \times\left[T_{n, m, \gamma}^{(1)}(x)+2 m T_{n, m-1, \gamma}(x)\right] \\
& +m(1+2 \gamma x) T_{n, m, \gamma}(x) .
\end{aligned}
$$

From the recurrence relation, it can be easily be verified that for all $x \in[0, \infty)$, we have $T_{n, m, \gamma}(x)=O\left(n^{-[(m+1) / 2]}\right)$.

Remark 2. From Lemma 1, using Cauchy-Schwarz inequality, it follows that

$$
B_{n, \gamma}(|t-x|, x) \leq\left[B_{n, \gamma}\left((t-x)^{2}, x\right)\right]^{1 / 2} \leq \sqrt{\frac{2 x(1+\gamma x)}{n-\gamma}} .
$$

Lemma 3. Let $x \in(0, \infty)$ and $W_{n, \gamma}(x, t)$ be the kernel defined in (1). Then for $n$ being sufficiently large, one has

(a) $\phi_{n, \gamma}(x, y)=\int_{0}^{y} W_{n, \gamma}(x, t) d t \leq 2 x(1+\gamma x) /(n-$ $\gamma)(x-y)^{2}, 0 \leq y<x$.

(b) $1-\phi_{n, \gamma}(x, z)=\int_{z}^{\infty} W_{n, \gamma}(x, t) d t \leq 2 x(1+\gamma x) /(n-$ $\gamma)(z-x)^{2}, x<z<\infty$.
Proof. First we prove (a); by using Lemma 1, we have

$$
\begin{aligned}
\int_{0}^{y} W_{n, \gamma}(x, t) d t & \leq \int_{0}^{y} \frac{(x-t)^{2}}{(x-y)^{2}} W_{n, \gamma}(x, t) d t \\
& \leq(x-y)^{-2} T_{n, 2, \gamma}(x) \\
& \leq \frac{2 x(1+\gamma x)}{(n-\gamma)(x-y)^{2}} .
\end{aligned}
$$

The proof of (b) is similar; we omit the details.

Theorem 4. Let $f \in D B_{r}(0, \infty), r \in \mathbb{N}$, and $x \in(0, \infty)$. Then for $n$ being sufficiently large, we have

$$
\begin{aligned}
\mid B_{n, \gamma} & (f, x)-f(x) \mid \\
\leq & \frac{2(1+\gamma x)}{n-\gamma} \sum_{k=1}^{[\sqrt{n}]} \bigvee_{x-x / k}^{x+x / k}\left(\left(f^{\prime}\right)_{x}\right) \\
& +\frac{x}{\sqrt{n}} \bigvee_{x-x / \sqrt{n}}^{x+x / \sqrt{n}}\left(\left(f^{\prime}\right)_{x}\right)+\frac{2(1+\gamma x)}{(n-\gamma) x} \\
& \times\left[\left|f(2 x)-f(x)-x f^{\prime}\left(x^{+}\right)\right|+|f(x)|\right] \\
& +\sqrt{\frac{2 x(1+\gamma x)}{n-\gamma}}\left[M 2^{r} O\left(n^{-r / 2}\right)+\left|f^{\prime}\left(x^{+}\right)\right|\right] \\
& +\sqrt{\frac{x(1+\gamma x)}{2(n-\gamma)}}\left|f^{\prime}\left(x^{+}\right)-f^{\prime}\left(x^{-}\right)\right|,
\end{aligned}
$$

where the auxiliary function $f_{x}$ is given by

$$
f_{x}(t)= \begin{cases}f(t)-f\left(x^{-}\right), & 0 \leq t<x \\ 0, & t=x \\ f(t)-f\left(x^{+}\right), & x<t<\infty .\end{cases}
$$

$\bigvee_{a}^{b} f(x)$ denotes the total variation of $f_{x}$ on $[a, b]$.

Proof. By the application of mean value theorem, we have

$$
\begin{aligned}
B_{n, \gamma}(f, x)-f(x) & =\int_{0}^{\infty} W_{n, \gamma}(x, t)(f(t)-f(x)) d t \\
& =\int_{0}^{\infty} \int_{x}^{t} W_{n, \gamma}(x, t)\left(f^{\prime}(u) d u\right) d t .
\end{aligned}
$$

Also, using the identity

$$
\begin{aligned}
f^{\prime}(u)= & \frac{f^{\prime}\left(x^{+}\right)+f^{\prime}\left(x^{-}\right)}{2}+\left(f^{\prime}\right)_{x}(u) \\
& +\frac{f^{\prime}\left(x^{+}\right)-f^{\prime}\left(x^{-}\right)}{2} \operatorname{sgn}(u-x) \\
& +\left[f^{\prime}(x)-\frac{f^{\prime}\left(x^{+}\right)+f^{\prime}\left(x^{-}\right)}{2}\right] \chi_{x}(u),
\end{aligned}
$$


where

$$
\chi_{x}(u)= \begin{cases}1, & u=x \\ 0, & u \neq x\end{cases}
$$

we can see that

$$
\begin{aligned}
& \int_{0}^{\infty}\left(\int_{x}^{t}\left(f^{\prime}(x)-\frac{f^{\prime}\left(x^{+}\right)+f^{\prime}\left(x^{-}\right)}{2}\right) \chi_{x}(u) d u\right) \\
& \quad \times W_{n, \gamma}(t, x) d t=0 .
\end{aligned}
$$

Also,

$$
\begin{aligned}
& \int_{0}^{\infty}\left(\int_{x}^{t}\left(\frac{f^{\prime}\left(x^{+}\right)-f^{\prime}\left(x^{-}\right)}{2}\right) \operatorname{sgn}(u-x) d u\right) W_{n, \gamma}(t, x) d t \\
& =\frac{f^{\prime}\left(x^{+}\right)-f^{\prime}\left(x^{-}\right)}{2} B_{n, \gamma}(|t-x|, x), \\
& \int_{0}^{\infty}\left(\int_{x}^{t}\left(\frac{f^{\prime}\left(x^{+}\right)+f^{\prime}\left(x^{-}\right)}{2}\right) d u\right) W_{n, \gamma}(t, x) d t \\
& =\frac{f^{\prime}\left(x^{+}\right)+f^{\prime}\left(x^{-}\right)}{2} B_{n, \gamma}(t-x, x) .
\end{aligned}
$$

Substitute value of $f^{\prime}(u)$ from (12) in (11) and using (14) and (15), we get

$$
\begin{aligned}
\left|B_{n, \gamma}(f, x)-f(x)\right| \leq & \left|\int_{x}^{\infty}\left(\int_{x}^{t}\left(f^{\prime}\right)_{x}(u) d u\right) W_{n, \gamma}(x, t) d t\right| \\
& +\left|\int_{0}^{x}\left(\int_{x}^{t}\left(f^{\prime}\right)_{x}(u) d u\right) W_{n, \gamma}(x, t) d t\right| \\
& +\frac{\left|f^{\prime}\left(x^{+}\right)-f^{\prime}\left(x^{-}\right)\right|}{2} B_{n, \gamma}(|t-x|, x) \\
& +\frac{\left|f^{\prime}\left(x^{+}\right)+f^{\prime}\left(x^{-}\right)\right|}{2} B_{n, \gamma}(t-x, x) .
\end{aligned}
$$

Using Lemma 1 and Remark 2, we obtain

$$
\begin{aligned}
\left|B_{n, \gamma}(f, x)-f(x)\right| \leq & \left|\int_{x}^{\infty}\left(\int_{x}^{t}\left(f^{\prime}\right)_{x}(u) d u\right) W_{n, \gamma}(x, t) d t\right| \\
& +\left|\int_{0}^{x}\left(\int_{x}^{t}\left(f^{\prime}\right)_{x}(u) d u\right) W_{n, \gamma}(x, t) d t\right| \\
& +\frac{\left|f^{\prime}\left(x^{+}\right)-f^{\prime}\left(x^{-}\right)\right|}{2} \sqrt{\frac{2 x(1+\gamma x)}{n-\gamma}} \\
= & \left|P_{n, \gamma}(f, x)\right|+\left|Q_{n, \gamma}(f, x)\right| \\
& +\frac{\left|f^{\prime}\left(x^{+}\right)-f^{\prime}\left(x^{-}\right)\right|}{2} \sqrt{\frac{2 x(1+\gamma x)}{n-\gamma} .}
\end{aligned}
$$

On applying Lemma 3 with $y=x-x / \sqrt{n}$ and integrating by parts, we have

$$
\begin{aligned}
& \left|Q_{n, \gamma}(f, x)\right|=\left|\int_{0}^{x} \int_{x}^{t}\left(f^{\prime}\right)_{x}(u) d u d t\left(\phi_{n, \gamma}(x, y)\right)\right| \\
& =\left|\int_{0}^{x} \phi_{n, \gamma}(x, y)\left(f^{\prime}\right)_{x}(t) d t\right| \\
& \leq\left(\int_{0}^{y}+\int_{y}^{x}\right)\left|\left(f^{\prime}\right)_{x}(t)\right|\left|\phi_{n, \gamma}(x, t)\right| d t \\
& \leq \frac{2 x(1+\gamma x)}{n-\gamma} \int_{0}^{y} \bigvee_{t}^{x}\left(\left(f^{\prime}\right)_{x}\right) \frac{1}{(x-t)^{2}} d t \\
& +\int_{y}^{x} \bigvee_{t}^{x}\left(\left(f^{\prime}\right)_{x}\right) d t \\
& \leq \frac{2 x(1+\gamma x)}{n-\gamma} \int_{0}^{y} \bigvee_{t}^{x}\left(\left(f^{\prime}\right)_{x}\right) \frac{1}{(x-t)^{2}} d t \\
& +\frac{x}{\sqrt{n}} \bigvee_{x-x / \sqrt{n}}^{x}\left(\left(f^{\prime}\right)_{x}\right) \\
& =\frac{2 x(1+\gamma x)}{n-\gamma} \int_{1}^{\sqrt{n}} \bigvee_{x-x / u}^{x}\left(\left(f^{\prime}\right)_{x}\right) d u \\
& +\frac{x}{\sqrt{n}} \bigvee_{x-x / \sqrt{n}}^{x}\left(\left(f^{\prime}\right)_{x}\right) \\
& \leq \frac{2 x(1+\gamma x)}{n-\gamma} \sum_{k=1}^{[\sqrt{n}]} \bigvee_{x-x / k}^{x}\left(\left(f^{\prime}\right)_{x}\right) \\
& +\frac{x}{\sqrt{n}} \bigvee_{x-x / \sqrt{n}}^{x}\left(\left(f^{\prime}\right)_{x}\right)
\end{aligned}
$$

where $u=x /(x-t)$.

On the other hand, we have

$$
\begin{aligned}
& \left|P_{n, \gamma}(f, x)\right| \\
& =\left|\int_{x}^{\infty}\left(\int_{x}^{t}\left(f^{\prime}\right)_{x}(u) d u\right) W_{n, \gamma}(x, t) d t\right| \\
& =\mid \int_{2 x}^{\infty}\left(\int_{x}^{t}\left(f^{\prime}\right)_{x}(u) d u\right) W_{n, \gamma}(x, t) d t \\
& \quad+\int_{x}^{2 x}\left(\int_{x}^{t}\left(f^{\prime}\right)_{x}(u) d u\right) d t\left(1-\phi_{n, \gamma}(x, t)\right) \mid \\
& \leq\left|\int_{2 x}^{\infty}(f(t)-f(x)) W_{n, \gamma}(x, t) d t\right| \\
& \quad+\left|f^{\prime}\left(x^{+}\right)\right|\left|\int_{2 x}^{\infty}(t-x) W_{n, \gamma}(x, t) d t\right| \\
& \quad+\left|\int_{x}^{2 x}\left(f^{\prime}\right)_{x}(u) d u\right|\left|1-\phi_{x, \gamma}(x, 2 x)\right|
\end{aligned}
$$




$$
\begin{aligned}
& +\int_{x}^{2 x}\left|\left(f^{\prime}\right)_{x}(t)\right|\left|1-\phi_{x, \gamma}(x, t)\right| d t \\
& \leq\left[\frac{M}{x} \int_{2 x}^{\infty} W_{n, \gamma}(x, t) t^{r}|t-x| d t\right. \\
& \left.+\frac{|f(x)|}{x^{2}} \int_{2 x}^{\infty} W_{n, \gamma}(x, t)(t-x)^{2} d t\right] \\
& +\left[\left|f^{\prime}\left(x^{+}\right)\right| \int_{2 x}^{\infty} W_{n, \gamma}(x, t)|t-x| d t\right] \\
& +\frac{2(1+\gamma x)}{(n-\gamma) x}\left|f(2 x)-f(x)-x f^{\prime}\left(x^{+}\right)\right| \\
& +\frac{2(1+\gamma x)}{n-\gamma} \sum_{k=1}^{[\sqrt{n}]} \bigvee_{x}^{x+x / k}\left(\left(f^{\prime}\right)_{x}\right) \\
& +\frac{x}{\sqrt{n}} \bigvee_{x}^{x+x / \sqrt{n}}\left(\left(f^{\prime}\right)_{x}\right) \\
& =R_{n, \gamma}(f, x)+S_{n, \gamma}(f, x) \\
& +\frac{2(1+\gamma x)}{(n-\gamma) x}\left|f(2 x)-f(x)-x f^{\prime}\left(x^{+}\right)\right| \\
& +\frac{2(1+\gamma x)}{n-\gamma} \sum_{k=1}^{[\sqrt{n}]} \bigvee_{x}^{x+x / k}\left(\left(f^{\prime}\right)_{x}\right) \\
& +\frac{x}{\sqrt{n}} \bigvee_{x}^{x+x / \sqrt{n}}\left(\left(f^{\prime}\right)_{x}\right)
\end{aligned}
$$

Applying Holder's inequality, Remark 2, and Lemma 1, we have

$$
\begin{aligned}
R_{n, \gamma}(f, x) \leq & \frac{M}{x}\left(\int_{2 x}^{\infty} W_{n, \gamma}(x, t) t^{2 r} d t\right)^{1 / 2} \\
& \times\left(\int_{0}^{\infty} W_{n, \gamma}(x, t)(t-x)^{2} d t\right)^{1 / 2} \\
& +\frac{|f(x)|}{x^{2}} \int_{2 x}^{\infty} W_{n, \gamma}(x, t)(t-x)^{2} d t \\
\leq & M 2^{r} O\left(n^{-r / 2}\right) \sqrt{\frac{2 x(1+\gamma x)}{n-\gamma}} \\
& +|f(x)| \frac{2(1+\gamma x)}{(n-\gamma) x} .
\end{aligned}
$$

Also,

$$
\begin{aligned}
S_{n, \gamma}(f, x) & \leq\left|f\left(x^{+}\right)\right| \int_{0}^{\infty} W_{n}(t, x)|t-x| d t \\
& \leq\left|f\left(x^{+}\right)\right| \sqrt{\frac{2 x(1+\gamma x)}{n-\gamma}} .
\end{aligned}
$$

Combining the estimates (17)-(21), we get the desired results. This completes the proof of Theorem.

\section{Rate of Convergence for Stancu Type Generalization of $B_{n, \gamma}$}

In 1968, Stancu introduces Bernstein-Stancu operators in [10], a sequence of the linear positive operators depending on two parameters $\alpha$ and $\beta$ satisfying the condition $0 \leq \alpha \leq \beta$. Recently many researchers applied this approach to many operators; for details see [11-17]. For $f \in C[0, \infty)$, Stancu generalization of operators (1) is as follows:

$$
\begin{aligned}
B_{n, \gamma}^{\alpha, \beta}(f(t), x)= & \sum_{k=1}^{\infty} p_{n, k, \gamma}(x) \int_{0}^{\infty} b_{n, k, \gamma}(t) f\left(\frac{n t+\alpha}{n+\beta}\right) d t \\
& +p_{n, 0, \gamma}(x) f\left(\frac{\alpha}{n+\beta}\right) \\
= & \int_{0}^{\infty} W_{n, \gamma}(x, t) f\left(\frac{n t+\alpha}{n+\beta}\right) d t,
\end{aligned}
$$

where $p_{n, k, \gamma}(x), b_{n, k, \gamma}(x)$, and $W_{n, \gamma}(x, t)$ are as defined in (1).

Lemma 5 (see [18]). If we define the central moments, for every $m \in \mathbb{N}$ as

$$
\begin{aligned}
\mu_{n, m, \gamma}^{\alpha, \beta}(x)= & B_{n, \gamma}^{\alpha, \beta}\left((t-x)^{m}, x\right) \\
= & \sum_{k=1}^{\infty} p_{n, k, \gamma}(x) \int_{0}^{\infty} b_{n, k, \gamma}(t)\left(\frac{n t+\alpha}{n+\beta}-x\right)^{m} d t \\
& +p_{n, 0, \gamma}(x)\left(\frac{\alpha}{n+\beta}-x\right)^{m}
\end{aligned}
$$

then $\mu_{n, 0, \gamma}^{\alpha, \beta}(x)=1, \mu_{n, 1, \gamma}^{\alpha, \beta}(x)=(\alpha-\beta x) /(n+\beta)$, and

$$
\begin{aligned}
\mu_{n, 2, \gamma}^{\alpha, \beta}(x)= & \frac{\alpha^{2}}{(n+\beta)^{2}}+x \frac{\left(2 n^{2}-2 n \alpha \beta+2 \alpha \beta \gamma\right)}{(n+\beta)^{2}(n-\gamma)} \\
& +x^{2} \frac{\left(n \beta^{2}+2 n^{2} \gamma-\beta^{2} \gamma\right)}{(n+\beta)^{2}(n-\gamma)} .
\end{aligned}
$$

For $n>m$ we have the following recurrence relation:

$$
\begin{aligned}
(n- & \gamma m)(n+\beta) \mu_{n, m+1, \gamma}^{\alpha, \beta}(x) \\
= & n x(1+\gamma x)\left[\left(\mu_{n, m, \gamma}^{\alpha, \beta}\right)^{(1)}(x)+m \mu_{n, m-1, \gamma}^{\alpha, \beta}(x)\right] \\
& +\left[m n+n^{2} x-(2 \gamma m-n)(\alpha-(n+\beta) x)\right] \mu_{n, m, \gamma}^{\alpha, \beta}(x) \\
& +\left[m \gamma(n+\beta)\left(\frac{\alpha}{n+\beta}-x\right)^{2}-m n\left(\frac{\alpha}{n+\beta}-x\right)\right] \\
& \times \mu_{n, m-1, \gamma}^{\alpha, \beta}(x)
\end{aligned}
$$


From the recurrence relation, it can be easily verified that for all $x \in[0, \infty)$, we have $\mu_{n, m, \gamma}^{\alpha, \beta}(x)=O\left(n^{-[(m+1) / 2]}\right)$.

Remark 6. Observe that $B_{n, \gamma}^{\alpha, \beta}$ preserve constant functions but not linear functions. If $\alpha=\beta=0$ these operators reduce to the operators defined in (1). Notice that

$$
\begin{aligned}
\mu_{n, 2, \gamma}^{\alpha, \beta}(x)= & {\left[\frac{\beta^{2}}{\gamma(n+\beta)^{2}}+\frac{2 n^{2}}{(n+\beta)^{2}(n-\gamma)}\right] x(1+\gamma x) } \\
& +\frac{\beta[(2 \alpha+\beta) \gamma-n(\beta+2 \alpha \gamma)]}{\gamma(n+\beta)^{2}(n-\gamma)} x+\frac{\alpha^{2}}{(n+\beta)^{2}} .
\end{aligned}
$$

Remark 7. From Lemma 3, taking $n$ to be sufficiently large and $x \in(0, \infty)$, we observe that

$$
\mu_{n, 2, \gamma}^{\alpha, \beta}(x) \leq \frac{C x(1+\gamma x)}{n-\gamma}
$$

where $C$ is positive constant.

Remark 8. Applying the Cauchy-Schwarz inequality and keeping the same condition as in Remark 7 for $x, n$, and $C$, we derive from Lemma 5 that

$$
B_{n, \gamma}^{\alpha, \beta}(|t-x|, x) \leq\left[B_{n, \gamma}^{\alpha, \beta}\left((t-x)^{2}, x\right)\right]^{1 / 2} \leq \sqrt{\frac{C x(1+\gamma x)}{n-\gamma}} .
$$

Lemma 9. Let $x \in(0, \infty)$ and $W_{n, \gamma}(x, t)$ be the kernel defined in (1). Then for $n$ being sufficiently large, we have

(c) $\phi_{n, \gamma}(x, y)=\int_{0}^{y} W_{n, \gamma}(x, t) d t=C x(1+\gamma x) /(n-$ $\gamma)(x-y)^{2}, 0 \leq y<x$.

(d) $1-\phi_{n, \gamma}(x, z)=\int_{z}^{\infty} W_{n, \gamma}(x, t) d t=C x(1+\gamma x) /(n-$ $\gamma)(z-x)^{2}, x<z<\infty$.

The proof is the same as Lemma 3, thus we omit the details.

Theorem 10. Let $f \in D B_{r}(0, \infty), r \in \mathbb{N}$, and $x \in(0, \infty)$. Then for $n$ being sufficiently large, we have

$$
\begin{aligned}
\left|B_{n, \gamma}^{\alpha, \beta}(f, x)-f(x)\right| & \\
\leq & \frac{C(1+\gamma x)}{n-\gamma} \sum_{k=1}^{[\sqrt{n}]} \bigvee_{x-x / k}^{x+x / k}\left(\left(f^{\prime}\right)_{x}\right) \\
& +\frac{x}{\sqrt{n}} \bigvee_{x-x / \sqrt{n}}^{x+x / \sqrt{n}}\left(\left(f^{\prime}\right)_{x}\right)+\frac{C(1+\gamma x)}{(n-\gamma) x}
\end{aligned}
$$

$$
\begin{aligned}
& \times\left[\left|f(2 x)-f(x)-x f^{\prime}\left(x^{+}\right)\right|+|f(x)|\right] \\
& +\sqrt{\frac{C x(1+\gamma x)}{n-\gamma}} \\
& \times\left[M_{1} 2^{r} O\left(n^{-r / 2}\right)+\left|f^{\prime}\left(x^{+}\right)\right|\right] \\
& +\frac{1}{2} \sqrt{\frac{C x(1+\gamma x)}{(n-\gamma)}}\left|f^{\prime}\left(x^{+}\right)-f^{\prime}\left(x^{-}\right)\right| \\
& +\frac{1}{2}\left|f^{\prime}\left(x^{+}\right)+f^{\prime}\left(x^{-}\right)\right| \frac{|\alpha-\beta x|}{n+\beta},
\end{aligned}
$$

where the auxiliary functions $f_{x}$ and $\bigvee_{a}^{b} f(x)$ were defined in Theorem 4.

The proof of the above theorem follows along the lines of Theorem 4; thus we omit the details.

\section{Conflict of Interests}

The authors declare that there is no conflict of interests regarding the publication of this paper.

\section{Acknowledgments}

The authors are thankful to the anonymous referee for making valuable comments leading to the better presentation of the paper. Special thanks are due to Professor Dr. Abdelalim A. Elsadany, Editor of Journal of Difference Equations, for kind cooperation and smooth behavior during communication and for his efforts to send the reports of the paper timely.

\section{References}

[1] V. Gupta, "Approximation for modified Baskakov Durrmeyer type operators," The Rocky Mountain Journal of Mathematics, vol. 39, no. 3, pp. 825-841, 2009.

[2] V. Gupta, M. A. Noor, M. S. Beniwal, and M. K. Gupta, "On simultaneous approximation for certain Baskakov Durrmeyer type operators," Journal of Inequalities in Pure and Applied Mathematics, vol. 7, pp. 1-15, 2006.

[3] V. Gupta and P. N. Agrawal, "Rate of convergence for certain Baskakov Durrmeyer type operators," Annals of Oradea University, Fascicola Matematica, vol. 14, pp. 33-39, 2007.

[4] H. Karsli and E. Ibikli, "Convergence rate of a new Bezier variant of Chlodowsky operators to bounded variation functions," Journal of Computational and Applied Mathematics, vol. 212, no. 2, pp. 431-443, 2008.

[5] V. N. Mishra, H. H. Khan, K. Khatri, and L. N. Mishra, "Hypergeometric representation for Baskakov-Durrmeyer-Stancu type operators," Bulletin of Mathematical Analysis and Applications, vol. 5, no. 3, pp. 18-26, 2013.

[6] V. N. Mishra, K. Khatri, and L. N. Mishra, "On simultaneous approximation for Baskakov-Durrmeyer-Stancu type operators," Journal of Ultra Scientist of Physical Sciences, vol. 24, no. 3, pp. 567-577, 2012.

[7] H. H. Khan, Approximation of classes of function [Ph.D. thesis], AMU, Aligarh, India, 1974. 
[8] X. M. Zeng and F. C. Cheng, "On the rates of approximation of Bernstein type operators," Journal of Approximation Theory, vol. 109, no. 2, pp. 242-256, 2001.

[9] M. Mursaleen, V. Karakaya, M. Ertürk, and F. Gürsoy, "Weighted statistical convergence and its application to Korovkin type approximation theorem," Applied Mathematics and Computation, vol. 218, no. 18, pp. 9132-9137, 2012.

[10] D. D. Stancu, "Approximation of function by a new class of polynomial operators," Revue Roumaine de Mathématique Pures et Appliquées, vol. 3, no. 8, pp. 1173-1194, 1968.

[11] R. Yang, J. Xiong, and F. Cao, "Multivariate Stancu operators defined on a simplex," Applied Mathematics and Computation, vol. 138, no. 2-3, pp. 189-198, 2003.

[12] H. M. Srivastava, M. Mursaleen, and A. Khan, "Generalized equi-statistical convergence of positive linear operators and associated approximation theorems," Mathematical and Computer Modelling, vol. 55, no. 9-10, pp. 2040-2051, 2012.

[13] M. Mursaleen, A. Khan, H. M. Srivastava, and K. S. Nisar, "Operators constructed by means of $q$-Lagrange polynomials and A-statistical approximation," Applied Mathematics and Computation, vol. 219, no. 12, pp. 6911-6918, 2013.

[14] V. N. Mishra and P. Patel, "Approximation by the DurrmeyerBaskakov-Stancu operators," Lobachevskii Journal of Mathematics, vol. 34, no. 3, pp. 272-281, 2013.

[15] V. N. Mishra, K. Khatri, and L. N. Mishra, "Inverse result in simultaneous approximation by Baskakov-Durrmeyer-Stancu operators," Journal of Inequalities and Applications, vol. 2013, no. 586, 2013.

[16] V. N. Mishra and P. Patel, "Some approximation properties of modified jain-beta operators," Journal of Calculus of Variations, vol. 2013, Article ID 489249, 8 pages, 2013.

[17] V. N. Mishra, K. Khatri, and L. N. Mishra, "Statistical approximation by Kantorovich-type discrete q-Beta operators," Advances in Difference Equations, vol. 345, no. 1, 2013.

[18] V. N. Mishra and P. Patel, "On simultaneous approximation for generalized integral type," Baskakov Operator. Submitted for publication. 


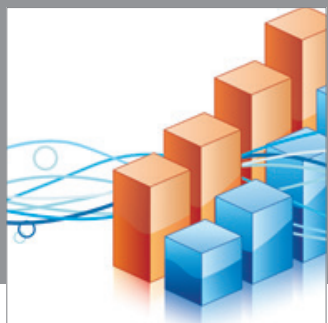

Advances in

Operations Research

mansans

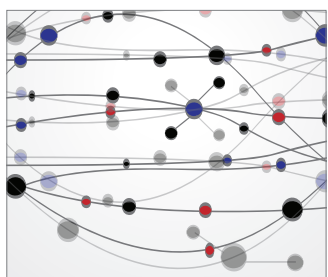

The Scientific World Journal
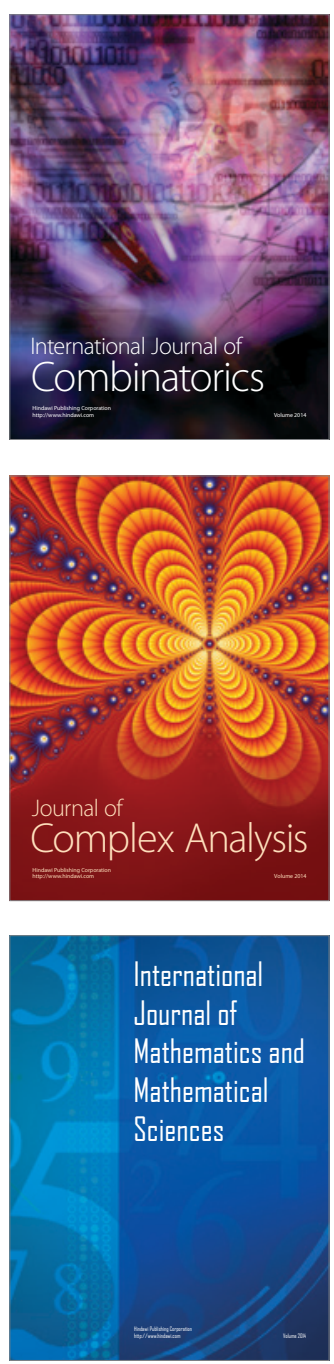
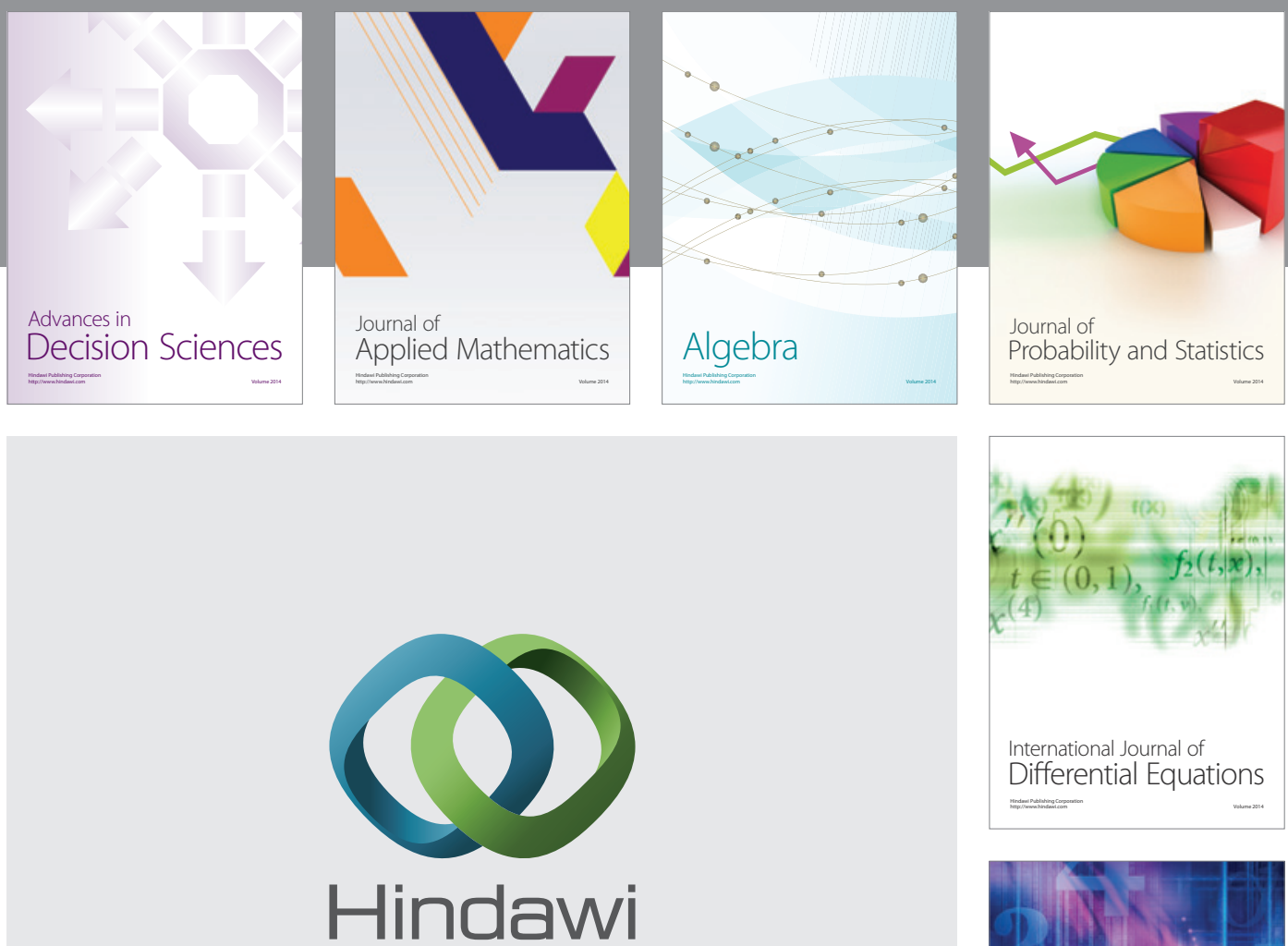

Submit your manuscripts at http://www.hindawi.com
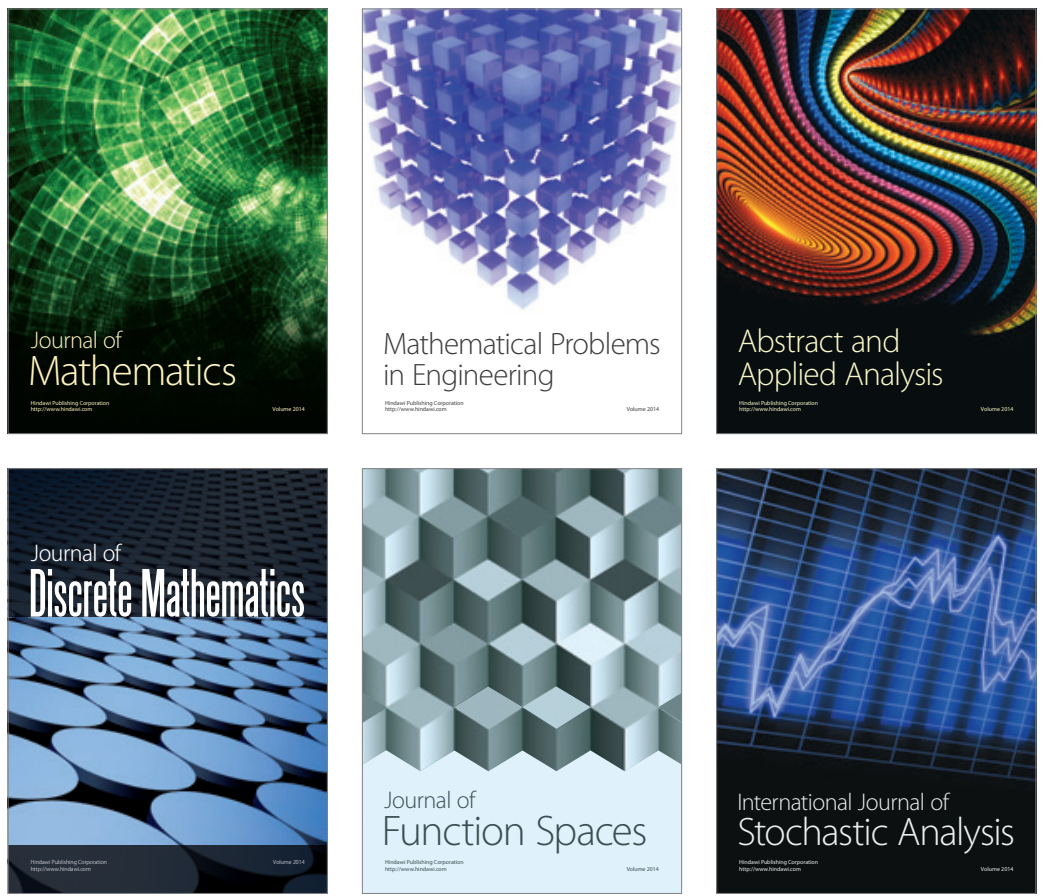

Journal of

Function Spaces

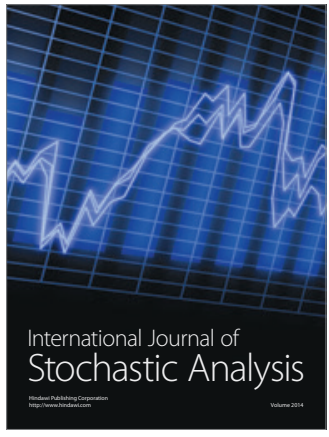

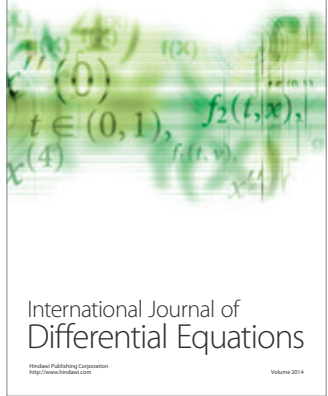
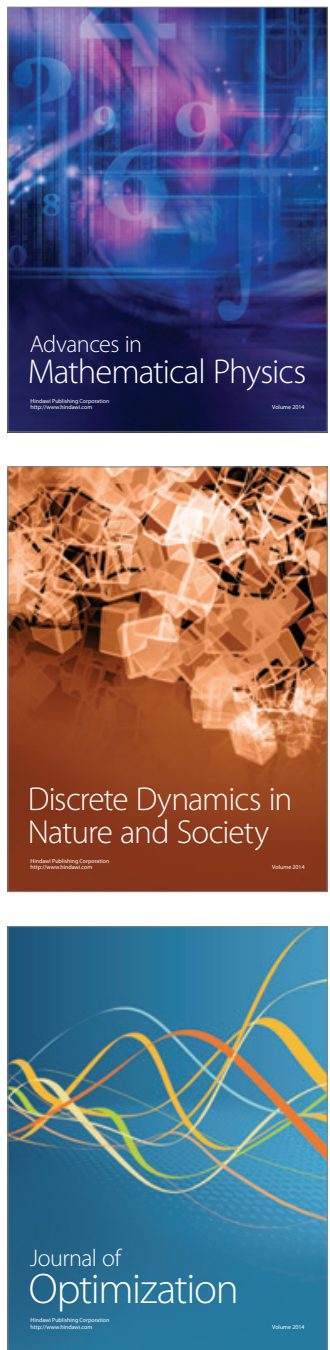